\title{
Algunas consideraciones en torno a los modelos de asentamientos con relación al marco natural: el ejemplo de la unidad biogeográfica murciano- almeriense durante el final del Paleolítico
}

\author{
Miguel Martinez Andreu
}

\section{INTRODUCCIÓN}

Una de las principales cuestiones que a nuestro juicio han de plantearse a la hora de valorar la interacción entre el hombre y el medio que le rodea, es el establecimiento de los limites de las unidades biogeográficas que componen el entramado sobre el que tienen lugar las distintas actividades.

La individualización de cada una de estas unidades requiere, sin embargo, una detallada evaluación de todos los rasgos que puedan perfilarse, de manera que en las conclusiones sobre una parcela geográfica determinada podamos reconocer que el conjunto de todas las diferencias que existen entre si son menores que respecto a otras áreas limitrofes.

En el caso del Sureste peninsular, su aparente unidad ha venido determinada más por razones históricas o políticas que por los verdaderos caracteres de los segmentos que lo integran. Esta realidad, no asumida en su justa medida por la Arqueologia, ha conducido a planteamientos muchas veces equivocados a la hora de estudiar el marco natural y sus influencias sobre una determinada manifestación cultural.

El objetivo que nos hemos propuesto a través de estas lineas probablemente no parte de apreciaciones rigurosas, ni tampoco pretende plantear 
un estado de la cuestión sobre el Paleolitico en esta parte de la geografia ibérica. Nuestro acercamiento es más bien una reflexión, una llamada de atención acerca de algunos aspectos, muchos de ellos sin aparente trascendencia, sobre esas implicaciones del medio físico en la adecuación de las estrategias de subsistencia, y de las ventajas que ello puedo tener a la hora de enfocar los estudios arqueológicos.

\section{LOS PRINCIPALES RASGOS DEL MEDIO FISICO. HACIA UNA DELIMITACIÓN DE LAS UNIDADES QUE INTEGRAN EL SURESTE PENINSULAR}

Dentro del territorio que comprende lo que geográficamente conocemos por Sureste se pueden distinguir varias series y ecosistemas (ALCARAZ 1986), que a su vez forman parte de otras tantas unidades biogeográficas. Básicamente pueden señalarse:

- La porción septentrional de Alicante, que pertenece a la denominada provincia Valenciano-Catalano-Provenzal-Balear

- La mayor parte de Albacete y algunas comarcas del norte de Murcia, que formarian parte de la provincia Castellano-MaestrazgoManchega.

- El sector más occidental de Almeria y una pequeña derivación hacia Albacete, que estaria integrado en la provincia Bética.

- El sur de Alicante, la mayor parte de Murcia y la porción suroriental de Almeria, que conforman la denominada provincia MurcianoAlmeriense, sobre la que incidiremos de manera especial.

\section{LA UNIDAD BIOGEOGRÁFICA MURCIANO-ALMERIENSE}

Se trata sin duda de una de las zonas más áridas de Europa, caracterizándose por la preponderancia de los ombroclimas árido y semiárido. Las precipitaciones medias anuales no suelen sobrepasar los $250 \mathrm{~mm}$, siendo casos extremos ciertos sectores de la costa y de las depresiones prelitorales. Sirvan como ejemplos los de Águilas (Murcia) y Cabo de Gata (Almeria), donde la alta evapotranspiración no puede ser compensada por las escasas precipitaciones $(173 \mathrm{~mm}$ en el observatorio de Águi- 
Algunas consideraciones en torno a los modelos de asentamientos...

las), y donde las necesidades reales para compensar ese efecto debieran estar en torno a los $1.000 \mathrm{~mm}$ de media anual (MONTIEL 1987). El saldo, en consecuencia, arroja un balance negativo cuyo resultado es un elevado déficit hídrico, constituyendo verdaderamente un caso extremo dentro de los márgenes del clima mediterráneo.

$\mathrm{Si}$ a todo ello unimos su diversificada geomorfologia, no ha de extrañar que los endemismos, dentro de las series que componen el paisaje vegetal, sean numerosos (ALCARAz 1986), destacando los íbero-africanos del sector almeriense. Se trata, en suma, de una fisionomia vegetal que denota una adaptación manifiesta a la escasez casi permanente de agua edáfica.

Pese a todo, la proximidad al mar atenúa los rigores de las temperaturas extremas, y los periodos cálidos, aunque prolongados, no resultan excesivamente intensos. Los inviernos son cortos y suaves, y los otoños mantienen temperaturas muy agradables. La porción comprendida entre la costa y las montañas del interior es la que acusa los mayores rasgos de aridez, sobre todo las depresiones de Lorca, Puerto Lumbreras y Fuente Álamo, mientras que las zonas más húmedas coinciden con los sectores montañosos que la separan de la Meseta, especialmente el suroeste de Albacete y la parte oriental de Granada y Almeria, pero como ya hemos señalado, estas áreas pertenecen ya a otras unidades biogeográficas.

\section{¿CONSTITUYEN LAS ACTUALES CONDICIONES UN HECHO CLIMÁTICO RECIENTE?}

Hemos de considerar que si la caracteristica climática más destacada es la acusada indigencia de precipitaciones, también lo es el carácter benigno y maritimo desde el punto de vista térmico. Esta última circunstancia hace de la región un lugar especialmente privilegiado, sobre todo durante los inviernos. Hay que reconocer, sin embargo, que estas ventajas térmicas se encuentran inmersas dentro de un medio natural con numerosos componentes hostiles al desarrollo de la actividad humana, especialmente los que se derivan de la naturaleza del suelo y los ocasionados por la ya apuntada problemática del agua (ANDRÉs 1986).

Las actuales condiciones del clima sólo permiten la existencia de un matorral camefítico muy ralo que constituye malezas predesérticas, con unos indices de cobertura muy bajos. 
En cuanto a las formas de relieve, muestran claramente las huellas de un morfoclima árido, con vertientes abruptas que se yerguen sobre amplias rampas de erosión o acumulación, guardando las formas externas una estrecha relación con la litologia, siendo manifiesta la erosión de tipo diferencial.

El estudio de algunos rasgos geomorfológicos (Montiel 1987, Ortiz 1986) y geológicos (FERNÁNDEZ 1986), indica que el sistema morfogenético árido se remonta a periodos muy antiguos, probablemente a partir del Neogeno. La existencia de formas de aplanamiento relativamente dilatadas, la escasa jerarquización de la red de avenamientos, con canales de escorrentia en paralelo que dan lugar a conos de deyección; redes con fuerte desarrollo en las cabeceras sobre materiales blandos que originan glacis, todo ello asi parece indicarlo.

Pero también los suelos son testimonio de la existencia casi permanente de climas áridos. La situación del complejo de cambio, la presencia de óxidos en los horizontes superficiales, los elevados contenidos en caliza activa, la abundancia de costras o concreciones salinas, son igualmente testimonio claro de su génesis árida.

En cuanto a la fauna fósil, todo parece indicar que durante el Mioceno superior se estaba agudizando proceso de cambio faunistico (AGusti et al. 1981; 1985), produciéndose una sustitución de la primitiva fauna de carácter forestal por otra de carácter más seco, representada mayoritariamente por inmigrantes orientales. Es precisamente en la etapa Turoliense cuando se constata ya una fauna de grandes herbivoros compuesta por équidos, bóvidos y ciervos, además de proboscideos y carnivoros felinos.

No tan alejados en el tiempo, y atendiendo sobre todo a los datos inferidos de los registros arqueológicos del Paleolitico superior, la aridez, aún siendo manifiesta, nunca llegó a los extremos que hoy conocemos. En ello habria que hacer notar el decisivo papel jugado por el hombre en tiempos más recientes sobre un medio natural mantenido en un precario equilibrio, incapaz de regenerarse.

Efectivamente, entre las especies capturadas por los grupos de cazadores y recolectores del final del Pleistoceno hay muestras inequivocas de que los bosques nunca alcanzaron un gran desarrollo, extremo éste que también viene confirmado por los análisis polínicos realizados en la Cueva del Caballo (Dupre 1989), sin embargo, junto a la nutrida representación de lagomorfos, también encontramos una pequeña representación de ciervos, bóvidos y con mayor peso especifico - probablemente en razón de la orografía del entorno- cabras; que si no constituyen elementos bioindicadores seguros, sí permiten al menos presumir la existencia 
Algunas consideraciones en torno a los modelos de asentamientos ...

de un paisaje no tan degradado como el actual. Con todo, hemos de admitir que en lo que venimos definiendo como unidad biogeográfica murciano-almeriense, se han dado unas condiciones climáticas muy peculiares desde finales del Terciario y que las pulsaciones frias del Pleistoceno nunca tuvieron en estas latitudes consecuencias climáticas drásticas, manteniéndose en esencia los márgenes atemperados del clima mediterráneo.

\section{LAS POSIBILIDADES DE EVALUACIÓN DE LOS MODELOS DE ASENTAMIENTOS CON RELACIÓN AL MARCO NATURAL. EL CASO DEL FINAL DEL PALEOLITICO}

Calcular de qué modo incidieron estos factores en las relaciones del hombre con el medio es una tarea dificil de abordar. No obstante, siguiendo algunas pistas que proporcionan los registros arqueológicos podriamos esbozar algunos rasgos. En primer lugar, haciendo un rastreo por la historiografía, nos encontramos con que algunos hechos Ilamaron la atención de los primeros investigadores que trabajaron en esta región. El caso de Louis SIRET (1893; 1931), pionero en la materia, puede ser elocuente cuando echaba en falta en las secuencias pertenecientes al Paleolitico superior una industria ósea así como cualquier tipo de manifestación artística, ya se tratara de arte mueble o parietal. Hoy sabemos que no es posible traspolar los mismos modelos que se encontraban en zonas geográficas alejadas, y que tal vez pudieron estar latentes en el pensamiento del autor belga, como el área franco cantábrica por ejemplo, y pretender encontrar una exacta correlación en el Sureste peninsular, pero también sabemos que por hoy los hallazgos de arte rupestre, a pesar de haber transcurrido casi cien años desde que Siret publicara sus trabajos, son realmente escasos, y no creemos que pueda achacarse sólo a la falta de prospecciones.

Deben existir, por lo tanto, razones que subyacen en el fondo de la cuestión y que aún no han sido desveladas, y son varias las preguntas que en este sentido pueden plantearse. Tal vez la primera sea de indole cultural, y uno de los argumentos que podrian esgrimirse contundente: ¿sencillamente por qué no usaron los mismos soportes? Sabemos por la Etnoarqueología que estos tipos de manifestaciones pueden revestir formas y técnicas muy diferentes, y quizá cabria la posibilidad de que se hubiesen plasmado sobre materias perecederas, o tal vez a través de pintura corporal; incluso que hayan desaparecido en gran parte como consecuencia de una conservación diferencial. De cualquier modo, la hipó- 
tesis de una conservación selectiva tan drástica no parece que tenga demasiado sustento, y por otro lado es innegable que en los escasos ejemplos de manifestaciones rupestres paleolíticas se percibe una clara preferencia por los lugares más sugerentes y ocultos. El ejemplo de la Cueva del Niño, en Ayna (Almagro Gorbea 1971), donde existen dos ambientes pictóricos distintos, uno exterior levantino, y otro en el interior, paleolítico, puede servir de ejemplo. Por otra parte, los más recientes hallazgos de la Cova Fosca y la Cova del Reinós, en Alicante (HERnANDEz 1988) parecen también indicar un tipo de emplazamientos más intimistas, si cabe la expresión, que aquellos más propios del denominado Arte Levantino, cuestión que evidentemente no resulta novedosa, pero que conviene tener presente si queremos dar algún sentido a esa escasez de manifestaciones paleoliticas en el Sureste.

No creemos que la respuesta a esta irritante duda se halle únicamente en las diferencias regionales, que sin duda las hay, sino más bien en una serie de fenómenos encadenados entre los que no debemos olvidar un hecho directamente relacionado con el medio físico, como es el menor desarrollo cárstico que el Sureste en general y la región Murciano-Almeriense en particular presentan. Se trataria en suma de un problema más cuantitativo que cualitativo, y si atendemos a las espectativas de hallazgos, parece lógico pensar que éstos serán inferiores en número allí donde las cavidades con tales caracteristicas sean también menores, y en último término, una cuestión que habrá de dirimirse mediante prospecciones bien planificadas.

En cuanto al arte mueble, tampoco creemos, a la vista de los hallazgos conocidos en áreas muy próximas, que esta porción del territorio peninsular constituya en absoluto una excepción. Los principales inconvenientes los encontrariamos, por un lado, en los pocos yacimientos que aún hoy han sido excavados con rigor, y de otro en los graves problemas de carbonatación que presentan la mayoria de sedimentos, envolviendo los materiales con una fina pelicula fuertemente adherida que impide reconocer cualquier traza, ya sean grabados o pigmentos, de no mediar un adecuado tratamiento de descarbonatación.

Pero si por ser prácticamente desconocido poco podemos valorar lo relativo al arte, afortunadamente sí contamos con otras evidencias proporcionadas por los registros arqueológicos que nos dan una información valiosa sobre la manera en que las estrategias de los cazadores y recolectores del final del Paleolitico sintonizaban con el medio físico que les rodeaba. Uno de los más claros exponentes sea tal vez el gran número de evidencias de lagomorfos en los yacimientos, cuestión que ha sido eje de no pocas polémicas acerca de su captura por parte del hombre, como 
también lo han sido los restos de aves y, sobre todo, los gasterópodos terrestres. Actualmente los estudios faunisticos, tanto en lo que respecta a conejos (Pérez RIPOLL 1980) como a caracoles (LoRENZo 1991) permiten asegurar que una buena parte de estas especies fueron objeto de atención. No obstante, la correcta evaluación del aporte alimenticio que constituyeron no oculta en ningún momento que su peso específico en la dieta, comparado con el rendimiento que proporcionan los mamíferos de medio y gran tamaño, es discreto. Pese a todo es innegable su importante carácter complementario en buena parte del Mediterráneo peninsular, probablemente más acentuado en las tierras bajas del Sureste, donde la aridez edáfica que al menos evocan los resultados polínicos (DUPRE 1989) propiciaría la existencia de grandes espacios abiertos. No sorprenden en este sentido, por ejemplo, las citas de la Cueva de la Palica (Serrón) por parte de J. Fortea (1970), que apunta: «... algo importante es la escasez industrial de La Palica y la abundancia de caracoles hasta proporciones ingentes"; o las nuestras en cuevas como Algarrobo, Caballo y Dentoles (MARtinez Andreu 1989), donde la densidad de gasterópodos de la misma especie y tamaño, varios miles en superficies no mayores de $6 \mathrm{~m}^{3}$, no sólo demuestra una selección, sino que descarta, ya que no podrian competir tantos individuos en espacios tan reducidos, cualquier tipo de deposición no intencionada. Su consumo aparece refrendado en la Cueva del Algarrobo, donde una de estas agrupaciones significativas, compuesta por unos treinta individuos de la especie lberus gualtierianus fue encontrada junto a un hogar.

Abundando en las referencias, las de Pericot (1942) en el Parpalló respecto a los lagomorfos (Lepus cuniculus) son elocuentes, como también, a pesar de no tener reflejo en el cómputo de evidencias, los gasterópodos terrestres (Helix), cuya abundancia es puesta de relieve por este autor.

Aún así, conviene no olvidar el escaso rendimiento que los helicidos proporcionan, cuya predación probablemente sólo estuvo compensada por su abundancia y fácil recogida y por ser un alimento nada despreciable desde el punto de vista bromatológico. Como ejemplo, señalar que de las casi 1.500 conchas de individuos adultos, en su mayor parte Iberus y en menor proporción Sphinterochila, recuperados en la Cueva del CabaIlo, el rendimiento de carne aprovechable apenas supera los $2 \mathrm{~kg}$ (LORENzo 1990).

Resulta pues evidente que estas actividades que podriamos calificar de complemento en la dieta sólo empiezan a cobrar sentido cuando unas y otras con contempladas en conjunto. Es entonces cuando podemos percibir que lagomorfos, aves, gasterópodos terrestres, frutos, y mariscos en las zonas costeras, podrian estar apuntando hacia ese tipo de economia 
que Flanery (1969) denominaba de amplio espectro, uno de cuyos más claros exponentes se encuentra en la malagueña Cueva de Nerja (JoRDA PARDO 1986).

Retomando el hilo de la cuestión, y tratando de buscar los elementos que particularizan los aspectos regionales propios de las tierras bajas del Sureste, habria que señalar que de admitir que esa aridez generalizada no constituye un hecho climático reciente, una parte del bagaje cultural y de las técnicas particulares de los grupos cazadores y recolectores que la poblaron estaria mediatizado por la mutua interacción con el medio a través de unos comportamientos especificos que deben ser distinguibles respecto a los puestos en práctica en otras regiones biogeográficas vecinas. Es cierto que la movilidad conllevaria el paso por zonas ambientales distintas, pero no es dificil creer que la predictibilidad y la propia concepción de subsistencia dejarian jugar un escaso papel a la aleatoriedad en los desplazamientos grupales. La apuesta por la existencia de territorios delimitados, así como de una adaptación ecológica concreta no está probada de forma incontrovertible, pero los datos que hoy podemos manejar si permiten asegurar que estos movimientos más o menos cíclicos por la costa del Sureste fueron lo suficientemente intensos como para hablar de una adecuación de las estrategias a un medio natural muy particular como es éste, y que necesariamente era bien conocido.

Tal vez, a tenor de los datos que proporcionan los registros arqueológicos, lo más singular de esa adaptación sea precisamente la falta de una gran especialización. Es evidente que aquí no podemos hablar de un control exhaustivo sobre una especie de alto rendimiento, como podrian ser el ciervo, la cabra, o en zonas del centro y norte de Europa el reno, durante el final del Paleolítico. Si hay un rasgo que se perfila como caracteristico éste es la diversificación, y en cierto modo no ha de extrañar que en estas latitudes lo específico y lo especial sean adjetivos un tanto faltos de contenido.

En un análisis a mayor escala no puede negarse un aire de familia común a todo el Mediterráneo peninsular, y en este sentido el Sureste no es una excepción, pero conforme nos acercamos a las áreas regionales que lo integran se van percibiendo los diferentes rasgos de personalidad. Asi, en el límite septentrional de lo que geográficamente venimos denominando provincia murciano-almeriense, nos encontrariamos con yacimientos como Cendres (VILLAVerde 1981) y Tossal de la Roca (CACHO 1988) que parecen marcar un punto de inflexión a nivel tecno-tipológico y también de tránsito geográfico hacia la vertiente levantina propiamente dicha; diferencias que se plasmarian para el caso del área que nos ocupa, en una menor leptolitización, un tratamiento más frágil en los instrumen- 
Algunas consideraciones en torno a los modelos de asentamientos ...

tos de borde rebajado, en especial las laminitas de finisimo retoque abrupto, y un menor porte en general de los útiles, que por lo demás siguen mostrando las mismas tendencias por grupos y, si cabe la expresión, esa envoltura de mediterraneidad que parece impregnar los cortejos industriales finipaleoliticos, a pesar de acusar más las diversidades regionales. Con todo, las semejanzas más notables, que trascienden sobre el plano tecnológico y se aproximan a los modelos económicos, se encuentran sin duda en las estaciones costeras andaluzas, de las que el ya reseñado yacimiento malagueño de Nerja es punto de referencia obligada.

De los rasgos que definen el Sureste, la disposición del relieve es uno de los que más han de tenerse en cuenta a la hora de valorar la distribución del poblamiento paleolitico. Los cordones montañosos paralelos a la costa marcan una gradual transición entre los ambientes áridos de ésta, carente por completo de cursos de agua permanentes, y las sierras del interior, más húmedas, que separan a la Meseta del Mediterráneo.

Esta disposición peculiar pudo favorecer una dirección de tránsito en sentido Norte-Sur, entre dos ambientes marcados por ritmos distintos. En la costa, los pocos lugares de afloramientos de agua, por un lado, y los cauces secos de las ramblas como perfectas vías de comunicación, por otro, parecen convertirse en los lugares más frecuentados. Las cavidades que participan en estas dos circunstancias son sin duda los lugares privilegiados en los que las ocupaciones pueden enfrentarse con éxito a un medio un tanto hostil, compensadas también por la garantia de recursos permanentes que el mar ofrece, y acaso por unas temperaturas agradables durante el invierno.

En los yacimientos que conocemos se detectan despliegues de actividades diversas, muchas de ellas marcadas por el entorno. Así, en los más próximos a la actual linea de costa, como Los Dentoles, o Los Boletes, se observa que una buena parte del tiempo se empleó en la provisión de mariscos, mientras que en los yacimientos intermedios esta actividad disminuye progresivamente en favor de la caza diversificada de mamiferos: cabra, caballo, uro, ciervo, nunca en grandes proporciones, junto a lagomorfos, aves, gasterópodos terrestres y algunos frutos, de los que al menos se tiene constancia de Vid silvestre (RIVERA-WALKER 1989). El caso de la Cueva del Caballo (MARtinez Andreu 1989), situada en la actualidad a 2,3 $\mathrm{km}$ del mar, parece mostrar un ejemplo de actividades costeras de repliegue; es decir, unas etapas de ocupación transitoria ya de espaldas al mar: un buen porcentaje de las evidencias marinas presentes se corresponden con especies de moluscos que ya fueron recogidos muertos y arrastrados por las mareas, con una finalidad en muchos 
casos suntuaria u ornamental; en cuanto a los comestibles, éstos constituyen sólo pequeñas reservas traidas con toda probabilidad desde la última estancia en línea de costa, y que se concretan en algunas decenas de moluscos y unos pocos peces que a lo sumo complementaron una dieta ya fundamentalmente continental.

Todo parece indicar que bien los ocupantes de este sitio no lo utilizaron como lugar de base desde donde operar la explotación intensa de los recursos marinos, lo que no deja de chocar teniendo en cuenta su distancia actual a la linea de costa; o el mar se encontraba lo suficientemente alejado como para hacer escasamente rentable el trayecto. También como lugar intermedio entre los tipos de asentamientos costeros se encuentra la Cueva del Algarrobo, hoy a poco más de $10 \mathrm{~km}$ de la playa. Esta mayor distancia hace que el peso especifico de este tipo de recursos sea ya verdaderamente insignificante. Algunos escafópodos y moluscos constituyen las únicas evidencias de consumo frente a un mayor repertorio de especies no aptas desde el punto de vista bromatológico, y que presentan perforaciones, en unos casos intencionadas y en otros aprovechando las casuales, muchas veces asociadas con un fin ornamental, como es el caso de las valvas de pecten, en las que es posible interpretar también otras finalidades domésticas más utilitaristas, hecho sobre el que ya reparó Pericot (1945) en El Parpalló y que parece confirmarse en los recientes trabajos de Soler (1990) sobre ese mismo yacimiento.

De cualquier modo, en El Algarrobo hay pruebas suficientes (LoRENZo 1991) de que estos ejemplares (de los que únicamente recogían las valvas derechas, más ahuecadas y por tanto con más posibilidades para usos), tal como se observa por la presencia de anélidos adheridos en las paredes internas, nunca fueron aprovechados como alimento.

Hay en suma una escasa actividad en lo concerniente a la pesca y el marisqueo que pone de manifiesto, todavía con más énfasis que en la Cueva del Caballo, esa decidida vocación de tránsito hacia el interior, en este caso hacia el Valle del Guadalentín, antesala de las sierras más húmedas del interior, que estos yacimientos tienen y que también queda patente en el origen de ciertas materias primas liticas, como el caso del silex jaspoide del Guadalentín, que fue traido en pequeñas porciones parcialmente descortezadas para la producción, casi exclusiva, de utillaje microlaminar.

Con todo, no parece que estos movimientos entre la costa y las áreas montañosas periféricas de la Meseta constituyan en si un rasgo diferenciador relevante, teniendo en cuenta que al fin y al cabo forman parte de un tipo de comportamiento común entre las sociedades cazadoras-reco- 
lectoras, ya suficientemente puesto de relieve en otras áreas geográficas vecinas (DAVIDSON 1989).

Tal vez la singularidad, si es que cabe la expresión, de esta unidad biogeográfica resida precisamente en ese cúmulo de pequeños matices, difíciles de percibir muchas veces, algunos de los cuales hemos ido entresacando a modo de ejemplos en las lineas que preceden.

Es cierto que muchas de las hipótesis que aqui se han planteado han de ser refrendadas. Otros aspectos, como los relativos a la aridez climática - no tanto la edáfica- que presumimos para esta región necesitan de un mayor contraste, y hasta es probable que a los ojos de una excesivamente compartimentada disciplina arqueológica, poco o nada tengan que ver el número de hallazgos de arte rupestre con los gasterópodos pulmonados, y éstos con las tanatocenosis costeras o con los fenómenos de carbonatación de los suelos; pero en el fondo, y ésta es la cuestión, todos ellos guardan una cierta relación tanto en cuanto constituyen variables con diverso grado de interdependencia. El clima y los suelos, la vegetación, la fauna, no mediatizan de una manera absoluta el comportamiento antrópico, que está regido también por la impredictibilidad de la conducta humana y por las pautas culturales, pero determina muchas de sus respuestas, y la explicación de no pocas interrogantes pasa, necesariamente, por una correcta evaluación de la interacción del hombre y del medio.

\section{BIBLIOGRAFIA}

Agusti, J. et al. (1981): "Casa del Acero, nueva fauna Turoliense de vertebrados (Mioceno superior de Fortuna, Murcia)", Int. del Instituto Paleontológico de Sabadell, XII, págs. 69-87. Sabadell.

Agusti, J. et al. (1985): "Nuevos datos sobre la bioestratigrafia continental de Murcia», Paleontologia i Evoluciò, XVIII. Sabadell.

AlCaraz, F. (1986): “El paisaje vegetal de Cartagena. Aspectos botánicos", Historia de Cartagena. Vol. I. págs. 212-240. Ediciones Mediterráneo. Murcia.

Almagro Gorbea, M. (1971): "La Cueva del Niño (Albacete) y la Cueva de La Griega (Segovia), dos yacimientos de arte rupestre recientemente descubiertos en la Peninsula Ibérica", Trabajos de Prehistoria, n. 28 , págs. 9-52. Madrid.

Andrés, J. L. (1986): «El ámbito terrestre», Historia de Cartagena. Vol. I, págs. 19-43. Ediciones Mediterráneo. Murcia

CACHO, C. (1988): "Tossal de la Roca. Vall d Alcalà (Marina Alta)", Memorias Arqueológicas de la Comunidad Valenciana. 1984-85, págs. 105-111. Valencia

Davioson, I. (1989): La economia del final del Paleolitico en la España Oriental. Trabajos Varios del S.I.P. n. ${ }^{\circ} 85,251$ págs. Valencia.

Dupre, M. (1989): "Informe polinico de la Cueva del Caballo", en Martinez, M., El Magdaleniense superior en la costa de Murcia, págs. 177-178. Editora Regional. Murcia. 
Fefinandez, J. C. (1986): "Sintesis geológica del Sureste español", Historia de Cartagena. Vol. I. págs. 47-112. Ed. Mediterráneo. Murcia.

FLANNERY, K. V. (1969): "Origins and ecological effects of early domestication in Iran and the Near East". págs. 73-100, en The domestication and explotaition of plants and animals. P. J. Ucko-G. Dimbleby (eds.) Aldine. Chicago.

ForteA, J. (1970): "La Cueva de La Palica, Serrón (Antas-Almeria). Avance al estudio del Epipaleolítico en el S. E. peninsular". Trabajos de Prehistoria, 28, págs. 61-97. Madrid.

Hernandez. M. S. Ferrer. P. y Catala, E. (1988): Arte rupestre en Alicante. Fundación Banco Exterior. Alicante.

Jok1: Pakid J. F. (ed.) (1986): La Prehistoria de la Cueva de Nerja (Málaga), 450 págs. Málaga.

LoRenzo, J. A. (1991): Informe de la malacofauna procedente de las cuevas del Caballo y Algarrobo (inédito).

Martinez Andeu, M. (1989): El Magdaleniense superior en la costa de Murcia. Editora Regional. Murcia.

MONTIEL, J. (1987): “Aproximación al medio bioclimático y los procesos morfológicos en Cabo Cope (Águilas)", Excavaciones y Prospecciones Arqueológicas. Serv. Reg. Patrimonio. Comunidad Autónoma, págs. 21-23. Murcia.

Ortiz, R. (1986): "Suelos", Historia de Cartagena. Vol. I, págs. 243-268. Ediciones Mediterráneo. Murcia.

Pérez Ripoll, M. (1980): “La fauna de vertebrados», en Marti, B. et al. Cova de l'Or (Beniarrés, Alicante). Vol. II. S.I.P. Trabajos Varios n. ${ }^{\circ} 65$, págs. 193-255.

Pericot, L. (1942): La Cueva del Parpalló (Gandia). Excavaciones del S.I.P. C.S.I.C. Instituto Diego Velázquez. Madrid.

Rivera, D. y WALkER, M. J. (1989): "A review of paleobotanical finding of early "vitis" ", in The Mediterranean and the origins of cultivated grape-vines. Review of Paleobotany and Palinology. N. 61. págs. 205-237. Amsterdam.

StRET, L. (1893): "L'Espagne Préhistorique", extrait de la Revue des Questions scientifiques, págs. 41-70. Bruselas.

- (1931): "Classification du Paléolithique dans le Sud-Est de 1 Espagne", XV Congrés International d'Anthropologie et d'Archéologie Prehistorique. Lisboa.

SOler MAYOR, B. (1990): "Estudio de los elementos ornamentales de la Cova del Parpalló", Saguntum 23, págs. 39-52. Valencia.

Villaverde, V. (1981): "El Magdaleniense de la Cova de les Cendres (Teulada, Alicante), y su aportación al conocimiento del Magdaleniense mediterráneo peninsular". Saguntum 16, págs. 10-35. Valencia. 